\title{
Virtual Tours and Representations of Cultural Heritage: Ethical Issues
}

\author{
Marco Ferrari \\ Lodovica Valetti
}

\section{Abstract}

Nowadays museums realities are increasingly interested in introducing advanced technological tools, in order to preserve and enhance the Cultural Heritage, as well as to make it more accessible and attractive. In fact, digital tools, such as Virtual Reality (VR) and Augmented Reality (AR), allow to provide effective responses to the needs of different types of users, to enhance the expressive qualities of the cultural asset, as well as to enrich the visitor's learning possibilities. However, the integration of new technologies in the management of Cultural Heritage involves different type of ethical issues. The paper analyses some of the ethical issues related to ontological, economic, and social aspects, trying to outline some principles able to limit these critical implications.

\section{Keywords}

museums, ethical issues, accessibility, virtual reality, augmented reality.

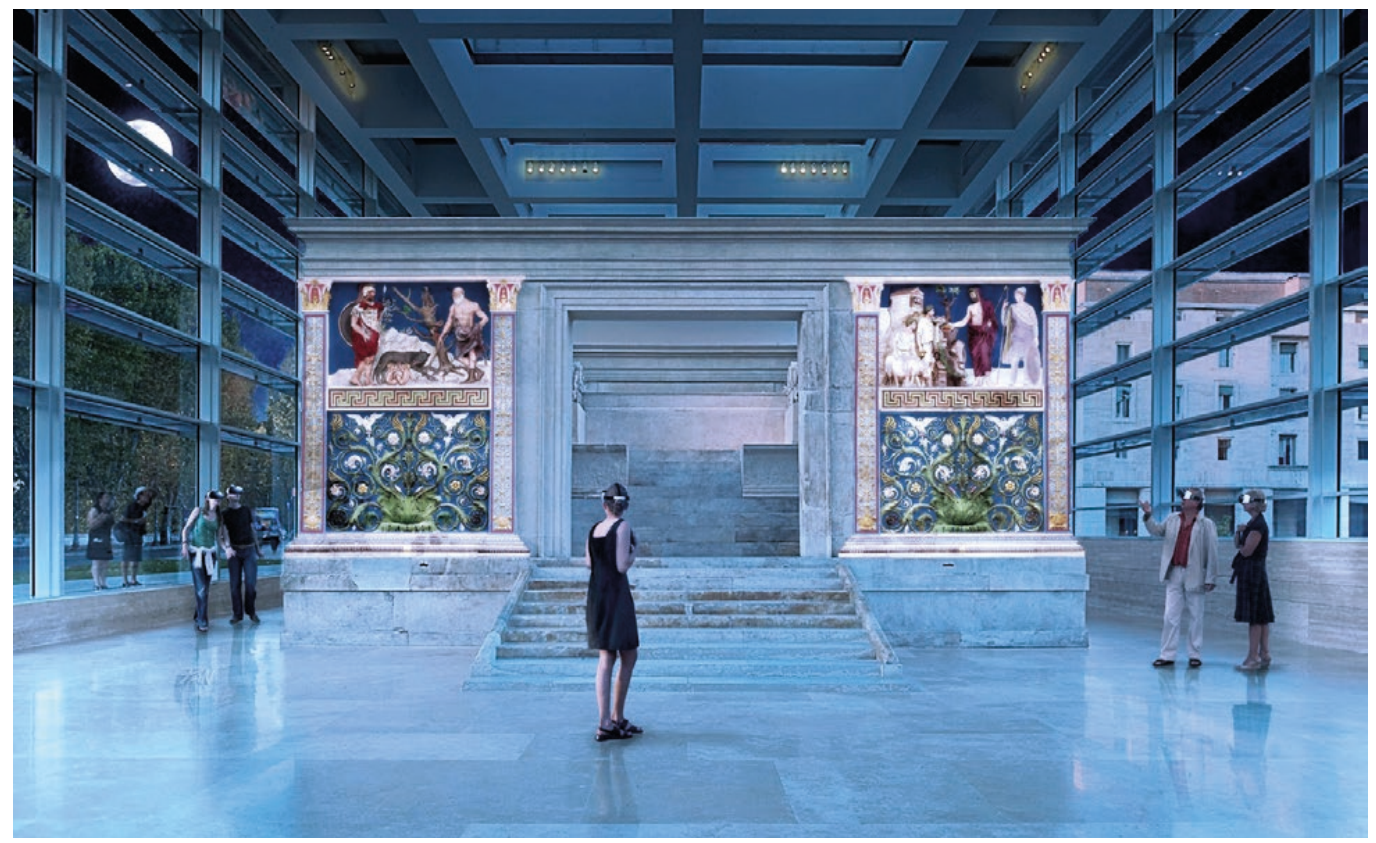




\section{Introduction: the Digital Fruition of the Cultural Heritage}

The contemporary indications concerning the protection and the enhancement of the Cultural Heritage promote the overcoming of the passive protection principles. Currently the insertion of purposes linked to the fruition and expansion of the communicative potential of the goods towards a wide and heterogeneous public are being promoted. As a consequence, in the last years innovative strategies have been introduced in the field of Cultural Heritage aimed at blending digital techniques and technological tools with conservation and enhancement strategies. The combination 'technological innovation - Cultural Heritage' opens new scenarios and requires the development of innovative approaches in the management of the Cultural Heritage. Within this frame, this paper investigates the application of Augmented Reality and Virtual Reality tools in historic sites and museums, considering the consequent ethical issues that this application involves.

Nowadays the accessibility and the correct communication of the Cultural Heritage are considered fundamental aspects of the enhancement strategies, aimed at making the cultural assets accessible to as many users as possible. One of the main approaches, applied in order to increase the involvement of the public, is the introduction in the field of Cultural Heritage of advanced digital technologies [Carrozzino, Bergamasco 20 I0, p. 452]. In fact, the enrichment of the tour experience in historical, archaeological and museum sites, combining traditional communication channels with new digital systems, based on multimedia contents (audio, video, hypertext, and/or three dimensionsal digital environments), is becoming increasingly popular [Orlandi et al. 20I4]. These strategies allow to provide effective responses to the needs of different types of users, to facilitate the enjoyment of the cultural assets, to enhance their expressive qualities, as well as to enrich the visitor's learning possibilities. In other words, new digital systems could contribute to the enhancement of the Cultural Heritage and its context, promoting its cultural and touristic values.

Currently, two of the most widespread technologies applied in the field of Cultural Heritage are the Virtual Reality (VR) and the Augmented Reality (AR). VR and AR are visual (the image is the centre of the communication) and interactive technologies (the active intervention of the users is required), which are characterized by providing immersive and interactive experiences. The introduction of these digital tools involves a change in the traditional learning process, no longer based on passive interaction, but rather on the active participation of users [Carrozzino \& Bergamasco 20 I0, p. 453]. In particular,Virtual Reality (VR) consists in the use of a computer technology that allows the virtual reconstruction of imaginary or historical sites (such as cities or sites belonging to past eras and no longer existing). Thanks to the application of VR, it is possible to create reconstructions that allow the visitor to visually perceive a site and its changes over time, as well as settings belonging to past eras. In a VR environment, users can have the perception of being totally immersed in an artificial world, interacting actively with it [Carrozzino \& Bergamasco 20 I0, p. 453] [Styliani et al. 2009, pp. 522-523]. A virtual reconstruction, if properly realized, can bring educational, historical, and scientific values. In addition to VR exhibitions, nowadays also the use of Augmented Reality (AR) is growing. $A R$ is a technology that allows to add digital contents to real exhibition scenarios, enriching the communication. The virtual information (i.e. $3 \mathrm{~d}$ objects, as well as any type of multimedia information, such as textual or pictorial data) is overplayed upon a video frames captured by a camera, giving the users the impression that the virtual cultural artefacts actually exist in the real environment [Styliani et al. 2009, p. 523].

The introduction of these kinds of technologies could have different purposes. First, they could supply a significant contribution in improving the communication. The tour experiences in historical, archaeological and museum sites could be enriched thanks to the introduction of multimedia contents, able to implement the visitor's learning possibilities through immersive experiences. The introduction of these technologies can be a way to increase the amount and the quality of information and to differentiate them by age and specific interests. The creation of innovative and amazing scenography (i.e. $3 \mathrm{~d}$ reconstructions) and the introduction of dynamic information transmission systems [Vaudetti et al. 20 I3, p. 95] could allow to increase the attractiveness the cultural goods for a wide audience, even non-specialists. 
In addition to the communicative one, there are other purposes, equally important. In some cases, the virtual reconstruction of the Cultural Heritage could allow the virtual access of spaces that currently cannot be opened to the public for security or conservation needs. Within this frame, the intervention would also be a trace of a contemporary operation on the heritage, in compliance with the restoration's guidelines of compatibility and minimal intervention. Furthermore, the need to preserve the historical-artistic memory of Cultural Heritage, constantly subject to degradation phenomena caused by natural and anthropic events (wars, terrorist attacks, etc.), is ever more urgent [Orlandi et al. 2014]. The digitalization of Cultural Heritage could be an opportunity for passing on historical memory to future generations. Moreover, the life cycle of Cultural Heritage could be extended by limiting the accessibility to the sites and promoting exclusively (or partially) their digital use.

\section{Ethical Issues}

As seen, the use of VR and $A R$, as tools for the protection, development, knowledge, and fruition of Cultural Heritage, on one hand, achieves numerous goals, collecting wide interest and success of the public; on the other hand, it raises some ethical questions, mainly related to the ontological, economic and social sphere.

The first question concerns authenticity. In the context of the reconstruction through VR and AR of a setting (fig. I), a monument, or an archaeological object, we must ask ourselves about the authenticity of reproduction. In the case of virtual restitution of an object or a context that presents multiple gaps, the result irremediably presents a character of subjectivity, although the choices can be supported undoubtedly by an in-depth historical and scientific investigation. The outcome is the product of a univocal interpretation of the past through one of the many possibilities of analysis. In a try to bring the visitor closer to an in-depth knowledge of an object, in reverse, there is the risk of turning it away from the original, devaluing its value [Styliani et al. 2009, p. 525]. Therefore, the boundary between reality and fiction arises as to the main theme in VR and AR use. The informative and playful dimension must not risk compromising the authenticity of the work.

Secondly, we must ask about the economic feature of this technology. Design, configuration, and maintenance of $V R$ and $A R$ systems usually have very high costs; their use often requires large spaces dedicated, and qualified personnel who take care of their operation and use by the public. Therefore, it is an instrument in the economic possibilities of wealthy museum realities, able to sustain the technological investment; realities already

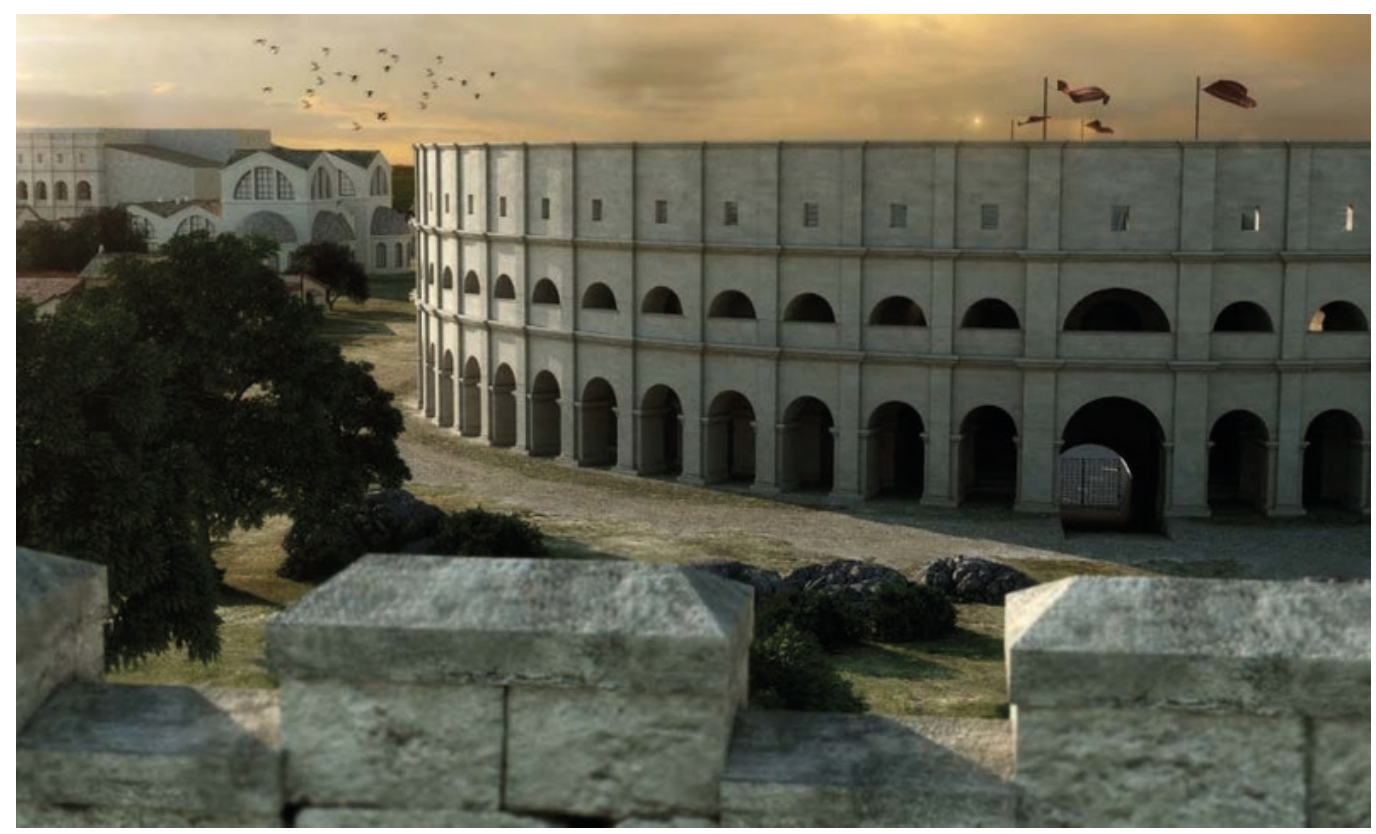


started and recognized, which - through their revenues or the support of patrons - implement the development of a heritage that may already be widely known, promoting its fruition in an innovative, diversified, modern, and endearing way. In the case of small, less fortunate museums, with reduced spaces and limited resources, the adoption of this technology, for example, would make accessible more objects normally not exposed due to lack of space, as well as it would offer more attractive visiting ways, welcoming broad public interest and obtaining profit and sustenance. An economic gap emerges in the potential of these instruments: they are promoted by already virtuous realities and hardly available to others that would need them to promote themselves.

Finally, a delicate question about social context arises. On one hand, the VR and AR use is extremely inclusive, providing opportunities to people with special needs (visual, acoustic, vocal, motor disabilities, and learning difficulties); however, on the other hand, the devices currently in use allow a type of visit characterized by a strong individualism. The isolation in the experience, often combined with the absence of a guide, can entail a loss of interest by the user: the deprivation of a stimulating relational exchange - between visitors or with an expert guide - risks to reduce the educational value of the contents offered as well as the indispensable emotional dimension, despite the strong visual impact of the context. Moreover, the growing diffusion and application of these technologies in daily and domestic life, from cinema to videogames, implying the possibility of seeing without imagining, can induce an estrangement of the user from the essence and the purpose of the experience itself: the significance of the artwork, the scientific value of the reconstruction, the informative and didactic goal. Therefore, we witness the feared «Guggenheim effect» [Carrozzino \& Bergamasco 20 I0, p. 457]: the fascination of the container overcomes and reduce the content, transforming the experience into mere entertainment rather than an opportunity to transmit information.

\section{Conclusions: Principles for Alternative Scenarios}

The use of these technologies, therefore, entails great advantages but also raises questions that lead us to reflect on principles able to limit their critical implications.

Firstly, the sources on which to build the virtual reading of the Cultural Property must be undeniable, as a guarantee and protection of its values of authenticity and uniqueness.

Secondly, from the point of view of communication, technology must act as a means of knowledge and culture, not as a mere attraction in order to implement tourist and economic flows. The main purpose should be the communication of historical, artistic, and cultural values of a population, as well as the authenticity and uniqueness of a work. Therefore, it is necessary a deep awareness based on education and culture: both on the part of the developers towards the contents to be communicated, and on the part of the users towards the added value that augmented reality offers. VR and AR are also instruments whose use must be calibrated and measured on the monument itself; cultural heritage institutions must assess their actual necessity and usefulness.

Concerning the economic gap that underlies the possibilities of each museum, we have to reconsider the criteria for access to funding and redefine the distribution of resources for the design and development of these technologies. In this way, less fortunate museums could benefit from funding for the conservation and enhancement of their heritage, provided that the proposals to be financed are examined by a multidisciplinary scientific committee that controls the methodological validity and the ethical implications.

Finally, technological development must increasingly be directed towards collective inclusiveness, preferring opportunities for comparison and evolving from the individual dimension. The correct communication of scientific contents must be guaranteed through the interaction with a guide or through the presence of an interface that can offer support for the visit, hoping that the technological innovation may soon lead to social and increasingly inclusive use.

In conclusion, VR and AR represent interesting tools to share knowledge, conservation, and development of the values of Cultural Heritage, provided that both their planning and fru- 
ition are based on awareness and respect for uniqueness, materiality, and cultural, historical, and artistic values. There must be rules that control their use informing users that these technologies are not games: they share contents and information of high cultural value. The relationship between man and machine and the concepts of real and virtual must be kept at the center of innovation and application in this field. The many opportunities and risks impose that society negotiates the uses of these technologies, guided and supported by a coherent philosophical approach [Arcagni 2016]. In this way, these tools will become part of a historical moment and will represent a cultural value of our century to be handed down.

\section{References}

Arcagni Simone (2016). L'etica della realtà virtuale. http://www.llsole24ore.com/art/tecnologie/20I6-05-09//-etica-realtavirtuale-15 1037.shtml?uuid=AD4sn7 (February 2021).

Carrozzino Marcello, Bergamasco Massimo (2010). Beyond virtual museums: Experiencing immersive virtual reality in real museum. In Journal of Cultural Heritage, I I, 20 I0, pp. 452-458.

Felline Francesca (20I5). Tecnologie innovative per la fruizione immersiva ed interattiva dei beni culturali. http://www. cniscintille.it/tecnologie-innovative-per-la-fruizione-immersiva-ed-interattiva-dei-beni-culturali/ (March 20।8).

Gabellone Francesco (2015). Integrated Technologies for Museum Communication and Interactive Apps in the PON DiCet Project. In: De Paolis Lucio Tommaso, Mongelli Antonio (eds.). Augmented and Virtual Reality. AVR 2015. Lecture Notes in Computer Science, 9254. Cham: Springer, pp. 3-16.

http://www.arapacis.it (May 202I).

http://www.eheritage.org/museum-installations/ (February 202I)

http://ec.europa.eu/research/social-sciences/index.cfm (February 202I).

http://www.progettoitacha.it/partner (February 202I).

http://www.researchers.polito.it/success_stories/progetti_metti_in_rete/nuove_tecnologie_per_le_collezioni_museali (February 2021).

Orlandi Marco, Vazzana Antonino, Zambruno Simone (2014). Tecnologia, Beni Culturali e Turismo: i TourVirtuali Virtual Tours) come strumento per una corretta comunicazione dei Beni Culturali. http://patrimonioculturale.unibo.it/sge/?page_id=829 (March 2018).

Styliani Sylaiou, Fotis Liarokapis, Kostas Kostsakis, Petros Patias (2009). Virtual museums, a survey and some issues for consideration. In Journal of Cultural Heritage, 10, 2009, pp. 520-528.

Vaudetti Marco, Minucciani Valeria, Canepa Simona (20I3). Mostrare l'archeologia - Per un manuale / atlante degli interventi di valorizzazione. Torino: Allemandi\&C.

\section{Authors}

Marco Ferrari, Dept. of Architecture and Design, Politecnico di Torino, marco_ferrari@polito.it

Lodovica Valetti, Dept. of Energy "Galileo Ferraris", Politecnico di Torino, lodovica.valetti@polito.it 
